\title{
eJRIEPS
}

Ejournal de la recherche sur l'intervention en éducation physique et sport

$35 \mid 2015$

Varia

\section{Analyse des phénomènes transpositifs dans l'enseignement du badminton à partir d'un programme épistémologique centré sur les curricula potentiels}

\section{Cédric Roure}

\author{
(2) OpenEdition \\ Journals \\ Édition électronique \\ URL : http://journals.openedition.org/ejrieps/1570 \\ DOI : 10.4000/ejrieps. 1570 \\ ISSN : 2105-0821 \\ Éditeur \\ ELLIADD
}

Référence électronique

Cédric Roure, «Analyse des phénomènes transpositifs dans l'enseignement du badminton à partir d'un programme épistémologique centré sur les curricula potentiels », eJRIEPS [En ligne], 35 | 2015, mis en ligne le 01 avril 2015, consulté le 03 octobre 2019. URL : http://journals.openedition.org/ ejrieps/1570 ; DOI : 10.4000/ejrieps.1570

a revue eJRIEPS est mise à disposition selon les termes de la Creative Commons Attribution 4.0 International License. 


\section{eJRIEPS 35 avril 2015}

\section{Analyse des phénomènes transpositifs dans l'enseignement du badminton à partir d'un programme épistémologique centré sur les curricula potentiels}

Cédric Roure

UFR Sport et EP de Brest, Centre de Recherche sur l'Education, les Apprentissages et la Didactique (CREAD - EA3875), France

\section{Résumé}

Partant du courant de recherche basé sur la théorie de l'action conjointe en didactique, l'objectif de cette étude est d'analyser les phénomènes transpositifs à l'œuvre dans l'enseignement en badminton. Prenant appui sur une analyse épistémologique des savoirs scolaires du badminton présents dans les curricula potentiels, nous confrontons cette analyse, considérée comme une lecture anticipatrice (Amade-Escot, 2013), à des données prélevées in situ lors d'un cycle de badminton sous l'angle des logiques chronogénétiques et mésogénétiques (Sensevy, 2007). Les résultats montrent une adéquation relative de la lecture anticipatrice avec les observations réelles qui peut être interprétée comme des décalages existants entre le programme épistémologique et le programme didactique (Schubauer-Leoni \& Leutenegger, 2005). Cette recherche permet ainsi d'approfondir les connaissances relatives à la part du processus de transposition didactique effectué par l'enseignant.

Mots-clés

TACD, épistémologie, éducation physique, transposition didactique, badminton, curricula potentiels

\section{Introduction}

Les recherches actuelles portées sur l'intervention, dans le cadre de la théorie de l'action conjointe en didactique (TACD), visent à rendre compte du fonctionnement des systèmes didactiques classiquement définis comme système ternaire d'interactions (Brousseau, 1998). Ce courant de recherche analyse l'activité en contexte des acteurs (enseignant et élèves) en s'appuyant particulièrement sur le concept de transposition didactique appréhendé in situ c'est-à-dire en relation avec la dialectique « contrat/milieu didactiques » (Amade-Escot, 2013). Considérant l'action conjointe du professeur et des élèves comme 


\section{eJRIEPS 35 avril 2015}

une coproduction de connaissances à la lumière de pratiques culturelles les légitimant, les chercheurs et l'auteur de cet article proposent d'étudier les phénomènes transpositifs en articulant deux programmes : le programme épistémologique et le programme didactique (Schubauer-Leoni \& Leutenegger, 2005). Le programme épistémologique a pour enjeu principal de saisir l'organisation des objets de savoirs et ses répercussions sur les phénomènes d'enseignement/apprentissage. Les recherches conduites dans cette direction tentent d'expliquer la structuration des savoirs à enseigner ou enseignés, pour reprendre les termes utilisés par Develay (1992), à partir de différentes échelles : les programmes scolaires, un établissement ou encore un professeur d'EPS. Le programme didactique se différencie de celui-ci dans la mesure où il cherche à saisir l'activité des sujets et des institutions et leurs rapports évolutifs aux objets de savoirs. Cette deuxième orientation fait référence à un ensemble de recherches visant à étudier l'activité des acteurs, enseignant et élèves, dans leur contexte d'intervention à la lumière d'actions conjointes. Désirant questionner les formes de transmission de la culture corporelle et sportive dans divers milieux (EPS, entraînement, éducation adaptée...), les chercheurs essayent de caractériser les processus conjoints d'élaboration des savoirs in situ. En définitive, cette approche didactique se donne pour visée d'éclairer la façon dont les individus participent conjointement à la diffusion et à l'appropriation des savoirs culturels au sein de diverses institutions.

Ces deux programmes sont considérés comme complémentaires lorsqu'ils interrogent les phénomènes transpositifs à l'œuvre dans l'enseignement du badminton que nous étudions ici. Adoptant une analyse ascendante de la transposition didactique selon le point de vue de l'enseignant, la TACD marque un tournant vis-à-vis des analyses descendantes formalisées antérieurement (Chevallard, 1985; Develay, 1992; Martinand, 1986). L'enjeu de cette posture théorique est d'articuler " délibérément les dimensions interactionnelle et épistémique des situations d'apprentissage "(Amade-Escot, 2013). En ce sens, le programme épistémologique s'intéresse à l'organisation des objets de savoirs et ses répercussions sur les phénomènes d'enseignement/apprentissage. II vise à déterminer quels sont les enjeux de savoirs mis à l'étude, ce qui lui confère une fonction épistémique et exploratoire. Afin de respecter les assises théoriques de la TACD, nous avons choisi de mener une analyse épistémologique sur les savoirs scolaires du badminton repérables au travers des curricula potentiels. Ces curricula sont entendus comme des propositions d'actions planifiées pour instruire en fonction d'un contexte donné, qui émanent des acteurs d'une discipline (Musard, Robin, Nachon \& Gréhaigne, 2008). Nous justifions ce 


\section{eJRIEPS 35 avril 2015}

choix par le fait qu'une analyse centrée directement sur des propositions de savoirs scolaires émanant des acteurs de l'enseignement est compatible avec une analyse ascendante des processus de transposition didactique centrés sur l'enseignant. Ainsi pensé, ce programme épistémologique, apparenté à une analyse a priori, constitue une grille de lecture anticipatrice pour le chercheur. Cette grille lui permet d'étudier l'univers des possibles et d'apprécier en quoi les situations d'apprentissage peuvent constituer un milieu favorable aux savoirs visés (Amade-Escot, 2013). Quant au programme didactique, son analyse des logiques d'actions conjointes des acteurs peut être considérée comme un révélateur des processus d'élaboration et de diffusion des objets de savoir en contexte d'intervention. Etant également en cohérence avec l'analyse ascendante des phénomènes transpositifs, ce programme fonctionne comme une analyse a posteriori révélatrice d'une dynamique, co-construite entre l'enseignant et les élèves ayant rapport à l'avancement des objets de savoir dans le temps et aux modifications du milieu utilisées pour apprendre. Notre objectif est donc de prendre appui sur un travail épistémologique existant centré sur les savoirs scolaires des sports de raquette en EPS (Roure, 2013) afin d'analyser les phénomènes transpositifs à l'œuvre dans l'enseignement du badminton. Nous désirons mettre en perspective un programme épistémologique, centré sur les curricula potentiels et autorisant une analyse a priori des enjeux de savoirs mis à l'étude, avec un programme didactique analysant l'action conjointe du professeur et des élèves. Ainsi considéré, nous présenterons dans le cadre théorique suivant, le programme épistémologique comme une lecture anticipatrice de l'enseignement du badminton et le programme didactique comme le révélateur des logiques de construction conjointes des objets de savoirs. Nous évoquerons ensuite la complémentarité de ces deux programmes et nous terminerons par la présentation de l'analyse épistémologique portée sur les savoirs scolaires du badminton afin d'envisager des hypothèses de recherche en lien avec l'enseignement de cette APSA.

\section{Cadre théorique de la recherche}

\subsection{Le programme épistémologique comme lecture anticipatrice}

Le programme épistémologique considéré comme une lecture anticipatrice constitue une grille de lecture pour le chercheur lorsque ce dernier désire s'appuyer sur la structuration des savoirs d'une discipline ou d'une APSA. Dans cette optique, l'appui sur l'épistémologie des savoirs permet de saisir les objets de savoirs fondamentaux, porteurs de la culture de l'APSA enseignée (Roure, 2013). Afin de saisir précisément les enjeux de la transmission de savoirs en badminton, nous nous appuyons sur un travail épistémologique existant à 


\section{eJRIEPS 35 avril 2015}

propos de cette activité (Roure, 2013). Cet auteur a élaboré un cadre théorique permettant de réaliser une analyse épistémologique des savoirs scolaires des sports de raquette à partir de l'étude des curricula potentiels repérés dans des publications professionnelles sur une période de 48 ans (1963 à 2011). Cette approche épistémologique, développée selon des angles historiques et sociologiques, nous intéresse dans la mesure où les résultats mettent en évidence une structuration des savoirs scolaires autour de concepts centraux à l'intérieur desquels sont déclinés des objets d'apprentissage (OA) et des mises en œuvre (MO) en lien avec des situations d'apprentissage (SA). Etant donné que nous sommes centrés sur l'enseignement actuel du badminton, nous exploiterons les résultats de cette recherche épistémologique seulement au niveau de la dernière période décrite par l'auteur, à savoir 1999 à 2011. La logique d'organisation des objets de savoirs, révélée au travers de ce programme épistémologique, s'organise autour de trois éléments : les OA, les $\mathrm{MO}$ et les concepts intégrateurs $(\mathrm{Cl})$. Les $\mathrm{OA}$ correspondent aux savoirs-faire ou autres habiletés motrices que l'élève doit acquérir, lesquels sont généralement envisagés dans leurs relations avec d'autres types de ressources à développer (e.g. connaissances déclaratives, connaissances procédurale, etc.). II peut ainsi s'agir de développer la maîtrise du coup droit ou du revers. Un OA apparaît le plus souvent encapsulé en une situation d'apprentissage ou dans une suite de situations d'apprentissage dont les contours sont spécifiés par les professeurs, en relation avec l'exposé de mises en œuvre (MO). Les MO sont relatives aux aménagements humains et/ou spatiaux ainsi que matériels. Un travail axé sur la maîtrise du coup droit peut ainsi être envisagé dans une logique coopérative, avec un relanceur produisant des volants aisés à renvoyer, sur un terrain comportant des zones cibles tracées, par exemple pour spécifier un enchaînement de frappes à produire. La SA correspondante peut ici viser à la réalisation d'un nombre d'échanges prédéterminé en vue de travailler la précision et la flexibilité de la frappe en coup droit. Enfin, les $\mathrm{Cl}$ correspondent à des référents communicationnels clés partagés par les membres d'une communauté donnée, comme les professeurs d'EPS, que nous pouvons associer à des « noyaux durs » de l'enseignement d'une APSA (Develay, 1992) en tant qu'ils organisent et structurent un ensemble d'OA et de MO. Ainsi peut-il en aller de l'idée de «prise en compte de l'adversaire », comme illustration d'un Cl en badminton, qui renvoie implicitement à la prise d'informations durant l'échange, ainsi qu'à la mise en place d'un projet de jeu ou encore et notamment à la gestion d'un rapport de force. Un $\mathrm{Cl}$, ainsi considéré, a donc une fonction communicationnelle. II est en effet de nature à orienter un professeur vers une conception des contenus et de l'enseignement relatif à 


\section{eJRIEPS 35 avril 2015}

une APSA, concourant par-là même à utiliser certains OA et MO. L'utilisation de ce programme épistémologique pour notre recherche consistera principalement à situer les objets de savoirs à enseigner en badminton, sous la forme d'OA et de MO. Nous nous réfèrerons également aux $\mathrm{Cl}$ structurant l'enseignement de cette activité dans la mesure où leur portée communicationnelle devrait nous autoriser à les retrouver dans les discours du professeur lorsque celui-ci définit et régule ses situations d'apprentissage (Sensevy, 2007).

2.2. Le programme didactique comme révélateur des logiques chronogénétiques et mésogénétiques

Le programme didactique se donne pour objectif premier d'éclairer la façon dont les acteurs participent conjointement à la diffusion et à l'appropriation des savoirs culturels au sein de diverses institutions (Amade-Escot \& Loquet, 2010). A ce titre, il peut être considéré comme un révélateur des processus d'élaboration et de diffusion des objets de savoir en contexte d'intervention. Classiquement, les chercheurs en ce domaine mettent en avant trois caractéristiques de l'action conjointe du professeur et des élèves : 1) la progression des savoirs au fil du temps didactique (logique chronogénétique) ; 2) les modifications du milieu utilisées pour apprendre (logique mésogénétique) ; 3) l'évolution des responsabilités prises par le professeur et les élèves vis-à-vis des savoirs (logique topogénétique). Dans le cadre de notre étude, le programme didactique sera utilisé au niveau des logiques chronogénétiques et mésogénétiques. Nous n’avons pas retenu la logique topogénétique car la lecture anticipatrice permise par le programme épistémologique ne nous permet pas de disposer d'informations relatives aux responsabilités prises par le professeur et les élèves. Selon l'axe chronogénétique, l'accent est porté sur l'introduction des objets de savoirs, supports de l'enseignement du badminton, et sur leurs logiques d'évolution au sein des séances et au fil du cycle. L'objectif est de saisir les savoirs enseignés par le professeur, leur découpage dans le temps ainsi que leur structuration à l'échelle d'un projet d'enseignement. Dans l'optique d'assurer une cohérence vis-à-vis du programme épistémologique, les savoirs enseignés par le professeur d'EPS en badminton seront catégorisés en OA et MO Cet axe chronogénétique sera principalement mis en relation avec le travail de définition des SA réalisé par le professeur (Sensevy, Mercier \& Schubauer-Leoni, 2000). Nous chercherons donc à identifier les $\mathrm{OA}$ et MO lorsque le professeur explique les SA aux élèves, notamment au travers des objectifs, buts et variables employées pour décrire les attentes de l'enseignant envers les élèves. Le deuxième axe employé dans notre étude 


\section{eJRIEPS 35 avril 2015}

(mésogénétique) sera centré sur une analyse des MO utilisés par le professeur au sein de ses interactions avec les élèves vis-à-vis des objets de savoirs mis à l'étude. L'objectif sera de comprendre dans quelle mesure l'introduction des MO permet à l'enseignant de structurer son discours et ses interactions avec les élèves dans une optique d'apprentissage. La poursuite de ce deuxième axe passera par une analyse des discours de l'enseignant lorsque celui-ci régule l'activité des élèves au sein des SA (Sensevy \& Co, 2000).

\subsection{Quelle complémentarité entre les deux programmes ?}

Cette recherche s'appuie sur les descripteurs utilisés dans la TACD afin de confronter les données issues de la lecture anticipatrice permise par l'analyse épistémologique et les données issues de l'observation d'un cycle d'EPS basé sur l'enseignement du badminton au lycée. Plus précisément, deux types de données supportent cette étude. Premièrement, des données relatives aux $\mathrm{OA}$, aux $\mathrm{MO}$ ainsi qu'aux mots clés structurant les discours des enseignants sont établies à partir des travaux épistémologiques menés sur l'enseignement du badminton (Roure, 2013). L'appui sur un travail de recherche existant nous permet d'obtenir des catégories d'OA et MO principalement utilisées dans l'enseignement du badminton au travers des SA. Nous disposons également d'un ensemble de mots utilisés par les enseignants pour expliquer et justifier leurs SA, ces mots faisant référence à des concepts intégrateurs qui leur donnent une structure et une cohérence. Et deuxièmement, des données relatives aux dynamiques évolutives du temps didactique (chronogénèse) et du milieu (mésogénèse) sont prélevées lorsque le professeur définit et régule les situations d'apprentissage (Sensevy, 2007). Notre objectif est ici de saisir et comprendre, dans l'activité de définition de l'enseignant, l'organisation des SA afin de pouvoir les catégoriser sous la forme d'OA et de MO. Pour ce faire, nous nous appuierons principalement sur le discours du professeur lorsqu'il explique les objectifs et les buts de chaque situation aux élèves, mais également lorsqu'il met en place des variables didactiques. Ces trois descripteurs (objectifs, buts et variables) fournissent suffisamment d'informations au chercheur pour établir les objets de savoir mobilisés au sein des SA. (Loizon, Margnes \& Terrisse, 2008). En ce qui concerne l'activité de régulation de l'enseignant, nous étudierons ses interactions avec les élèves lorsque cellesci sont centrées sur les relations entre les activités motrices déployées par les élèves et l'aménagement du milieu mis en place par le professeur. Notre but est de saisir l'organisation de l'enseignant au niveau des interactions entre l'utilisation des MO, son discours et l'activité déployée par les élèves. En définitive, la dimension conjointe de la 


\section{eJRIEPS 35 avril 2015}

TACD est principalement appréhendée au travers de l'activité de l'enseignant. Ce choix, qui peut paraître réducteur vis-à-vis de ce cadre théorique, s'explique par notre objectif de recherche. En effet, notre volonté de mettre en perspective un programme épistémologique et un programme didactique nous conduit à envisager les phénomènes transpositifs prioritairement sous l'angle de l'enseignant et d'envisager l'activité des élèves uniquement de manière indirecte. Dans cette perspective, nous n'avons retenu que les descripteurs de la TACD qui permettaient de répondre directement à notre objectif de recherche, à savoir la chronogénèse et la mésogénèse en relation avec les actions du professeur, c'est-à-dire définir et réguler.

\subsection{L'analyse a priori de l'enseignement du badminton}

Comme nous l'avons évoqué précédemment, nous prenons appui sur un travail de recherche existant ayant produit une analyse épistémologique des savoirs scolaires du badminton en EPS. Cette lecture anticipatrice, au sens d'Amade-Escot (2013), doit nous permettre de situer les objets de savoir enseignés dans cette activité, sous la forme d'OA et de $\mathrm{MO}$, ces derniers étant structurés par l'intermédiaire de Cl représentant les « noyaux durs » de l'enseignement de cette APSA (Develay, 1992). Nous pouvons prendre appui sur la dernière période de l'enseignement des sports de raquette, qui s'étend de 1999 à 2011 selon Roure (2013), dans la mesure où l'organisation des objets de savoirs perdure très vraisemblablement aujourd'hui. Cette période se caractérise par un enseignement cherchant à développer une adaptation tactique chez les élèves basée sur la rupture de l'échange et la recherche de zones à viser. Dans ce cadre organisateur, les professeurs d'EPS valorisent les apprentissages tactiques en se centrant sur un projet de jeu prioritaire représenté par la rupture de l'échange. La rupture est poursuivie par le biais d'une adaptation au jeu de l'adversaire mise en œuvre au travers de zones à viser sur le terrain, ou au travers de thèmes de jeu représentant des moyens d'introduire des incertitudes liées au jeu. A titre d'illustration, les SA peuvent demander aux élèves de prendre en compte le déséquilibre initial de leur adversaire (un joueur est placé sur le côté du terrain au début de l'échange) pour mettre en œuvre un plan de jeu visant à terminer l'échange en trois frappes maximum. Dans cet exemple, l'OA tactique visé par l'enseignant est la rupture de l'échange au sein d'une MO utilisant un thème de jeu en relation avec l'incertitude spatiale (placement particulier de l'adversaire au départ du point). Une autre SA peut demander à l'élève de placer une zone bonus dans le terrain de l'adversaire qui lui rapportera trois points si le volant tombe à l'intérieur de la zone ou si le joueur adverse est présent dans cette zone et fait une faute. Dans ce cas, l'enseignant utilise un OA 


\section{eJRIEPS 35 avril 2015}

stratégique, relatif à l'observation des points forts et faibles de l'adversaire (pour placer judicieusement la zone dans le terrain adverse) avec une MO représentée par une zone à viser au sol. Ces deux illustrations de SA utilisées dans l'enseignement du badminton démontrent que le sens culturel du badminton est axé sur « la capacité réflexive des joueurs sur les plans tactiques et stratégiques pour faire face à des rapports de force différenciés » (Roure, 2013). Les objets de savoir ainsi décrits, appartenant à l'enseignement du badminton, sont structurés au sein de Cl relatifs à l'adaptation à l'adversaire, à la construction d'un projet tactique et la formation d'un joueur réfléchi (Roure, 2013). Ce sens culturel du badminton se retrouve au sein des derniers programmes officiels de l'EPS parus en 2010 pour les classes du lycée. Les compétences attendues sont toutes libellées selon une entrée tactique, rapportée à l'adaptation à l'adversaire et à la prise en compte des évolutions de l'état du rapport de force.

\subsection{Les hypothèses de recherche}

Nous nous attendons à retrouver, lors de l'analyse des processus de chronogénèse et de mésogénèse chez un enseignement d'EPS, la lecture anticipatrice donnée par l'analyse épistémologique portant sur l'enseignement du badminton. De ce fait nous devrions observer, dans un cycle de badminton au lycée, un enseignement prioritairement centré sur des apprentissages tactiques et stratégiques mis en œuvre au sein d'environnements éducatifs variés utilisant des zones et des thèmes de jeu. Selon l'axe chronogénétique, l'observation d'un cycle de badminton majoritairement élaboré selon des entrées tactiques et stratégiques est attendue avec des séances axées sur la construction de projets de jeu en lien avec l'adaptation à l'adversaire. Nous devrions également identifier certains OA techniques envisagés comme des points d'appui nécessaires au développement tactique des joueurs. Quant à l'axe mésogénétique, nous émettons l'hypothèse que l'enseignant utilise les aménagements matériels et humains (traduits sous la forme de MO dans notre étude) pour structurer ses interactions avec les élèves et faciliter l'acquisition des objets de savoirs. Nous pensons observer des liens entre l'utilisation des thèmes de jeu et des zones à viser et les interactions professeur - élèves centrées sur le rapport de force, l'adaptation aux tactiques adverses et les différentes prises d'informations.

L'existence d'un travail de recherche relatif aux sports de raquette en EPS (Roure, 2013) permet opportunément de disposer de repères en termes d'objets de savoirs et de situations d'apprentissage (sous la forme d'OA et de $\mathrm{MO}$ ) et en termes de discours employés dans la justification des SA (sous l'angle des concepts intégrateurs structurant 


\section{eJRIEPS 35 avril 2015}

les objets de savoirs). Cette référence à un travail existant favorise notre investigation dont la logique est présentée ci-après.

\section{Méthodologie}

La méthodologie repose sur l'observation d'un enseignant d'EPS lors d'un cycle de badminton de huit séances en lycée et sur l'utilisation d'un travail épistémologique publié sur l'enseignement des sports de raquette en EPS. Ces deux dimensions sont en étroite relation avec nos hypothèses de recherche dans la mesure où nous cherchons à confronter l'analyse permise par un programme épistémologique avec des données observées in situ dans le cadre d'un programme didactique (Schubauer-Leoni \& Leutenegger, 2005). En cohérence avec notre cadre théorique, notre méthodologie vise ainsi à obtenir deux types de données : celles acquises par la lecture anticipatrice du programme épistémologique et celles obtenues par le programme didactique révélateur des logiques chronogénétiques et mésogénétiques.

Selon l'axe épistémologique, l'appui sur un travail de recherche existant nous permet de disposer de données relatives aux objets de savoir et à leur logique de structuration pour l'enseignement du badminton. Sans reprendre l'ensemble des éléments développés lors du cadre théorique (point 2.4), nous rappelons que les objets de savoir à enseigner dans l'APSA badminton sont découpés sous la forme d'OA et de MO. Ces deux catégories ont été établies par Roure (2013) à partir d'analyses des curricula potentiels publiés dans une revue professionnelle (Revue EP\&S). De plus, la structuration de ces objets de savoir sur une période donnée s'appuie sur l'identification de $\mathrm{Cl}$ qui correspondent à des référents communicationnels clés partagés par les membres d'une communauté donnée, comme les professeurs d'EPS, que nous pouvons associer à des «noyaux durs » de l'enseignement d'une APSA (Develay, 1992) dans le sens où ils organisent et structurent un ensemble d'OA et de $\mathrm{MO}$. Ces $\mathrm{Cl}$ sont de nature à orienter un professeur vers une conception des contenus et de l'enseignement relatif au badminton, concourant par-là même à utiliser certains $\mathrm{OA}$ et MO. En définitive, nous disposons de trois types de données à partir de la lecture anticipatrice permise par la référence à un travail publié dans le cas des sports de raquette : les $\mathrm{OA}$, les $\mathrm{MO}$ et les $\mathrm{Cl}$.

Afin de pouvoir confronter ces premières données à des observations réalisées dans le contexte d'une classe d'EPS, nous devons mettre en place un dispositif de recueil de données pour l'observation d'un cycle de badminton réalisé par un professeur d'EPS au lycée. Le contexte éducatif support de l'étude est caractérisé par un enseignant non 


\section{eJRIEPS 35 avril 2015}

spécialiste de l'APSA possédant une expérience d'enseignement de 10 ans. II s'agit d'une classe de première scientifique composé de 29 élèves (16 garçons et 13 filles) caractérisés par l'enseignant titulaire, aux niveaux 1 et 2 des compétences attendues fixées par les programmes officiels. Ce deuxième type de données est relatif au programme didactique au travers des logiques chronogénétiques et mésogénétiques. Afin de saisir l'évolution des objets de savoir au fil du temps didactique (axe chronogénétique), nous nous sommes appuyés sur des grilles d'observation structurées sous la forme d'OA et de MO (pour rester en cohérence avec le programme épistémologique) afin de catégoriser l'ensemble des SA mises en place par le professeur pendant son cycle. Nous recourrons à une analyse catégorielle, telle que définie par Bardin (1977), selon une procédure par boites étant donné que les catégories sont déjà établies en référence au travail épistémologique. Ainsi esquissée, notre grille d'observation est composée de trois types d'OA possibles : techniques, tactiques et stratégiques, et de trois catégories de MO envisageables: aucun aménagement, zones et thèmes de jeu, chacune d'entre elles pouvant se subdiviser selon l'aménagement humain employé : seul, avec partenaire et avec adversaire.

Le tableau I ci-dessous permet d'avoir un aperçu de cette grille d'observation avec des exemples dans chacune des catégories.

Tableau I. Grille d'observation des SA mises en place par l'enseignant d'EPS en badminton

\begin{tabular}{|c|c|c|c|c|c|c|c|c|c|c|c|}
\hline \multicolumn{3}{|c|}{ Objet d'apprentissage (OA) } & \multicolumn{9}{|c|}{ Mise en œuvre (MO) } \\
\hline \multirow[b]{2}{*}{ Techniques } & \multirow[b]{2}{*}{ Tactiques } & \multirow[b]{2}{*}{ Stratégiques } & \multicolumn{3}{|c|}{ Aucun aménagement } & \multicolumn{3}{|c|}{ Zones } & \multicolumn{3}{|c|}{ Thèmes de jeu } \\
\hline & & & $\begin{array}{l}\text { Seul } \\
\text { (S) }\end{array}$ & $\begin{array}{c}\text { Avec } \\
\text { partenaire } \\
\text { (AP) }\end{array}$ & $\begin{array}{c}\text { Avec } \\
\text { adversaire } \\
\text { (ADV) }\end{array}$ & S & AP & ADV & $\mathrm{S}$ & AP & ADV \\
\hline $\begin{array}{c}\text { Smash, } \\
\text { placement } \\
\text { sous le } \\
\text { volant, } \\
\text { amorti, etc. }\end{array}$ & $\begin{array}{l}\text { Chercher la } \\
\text { rupture, varier } \\
\text { les } \\
\text { trajectoires, } \\
\text { etc. }\end{array}$ & $\begin{array}{l}\text { Observer les } \\
\text { points forts et } \\
\text { faibles } \\
\text { adverses, etc. }\end{array}$ & \multicolumn{3}{|c|}{$\begin{array}{l}\text { Aménagement minimum : terrain, } \\
\text { filet, raquettes et volants }\end{array}$} & \multicolumn{3}{|c|}{$\begin{array}{c}\text { Zones sur le } \\
\text { terrain (cibles } \\
\text { ou interdites), } \\
\text { pour le } \\
\text { placement d'un } \\
\text { joueur }\end{array}$} & \multicolumn{3}{|c|}{$\begin{array}{l}\text { Thèmes avec } \\
\text { incertitudes } \\
\text { spatiales, } \\
\text { temporelles ou } \\
\text { événementielles }\end{array}$} \\
\hline
\end{tabular}

Cette grille d'observation est accompagnée d'enregistrements vidéo afin de pouvoir utiliser les discours de l'enseignant lorsqu'il définit les SA (Sensevy, 2007). L'utilisation vidéoscopée s'est avérée indispensable pour coder correctement les SA sous la forme d'OA et de MO. Pour ce faire, nous avons sélectionné les extraits vidéos relatifs à l'activité 


\section{eJRIEPS 35 avril 2015}

de définition du professeur, en retenant trois éléments clés reconnus par la littérature scientifique pour identifier les objets de savoir mis à l'étude : les objectifs, les buts et les variables didactiques des SA (Loizon, Margnes \& Terrisse, 2008). Pour saisir les modifications du milieu utilisées pour apprendre (axe mésogénétique), notre recueil de données s'appuie sur une grille d'observation des MO utilisées par le professeur (telle que présentée précédemment) ainsi que sur des enregistrements vidéo des leçons. L'outil vidéo est de nouveau utilisé en sélectionnant uniquement les extraits où le professeur est dans une activité de régulation (Sensevy, 2007). Nous avons donc retenu tous les passages pendant lesquels le professeur intervient auprès des élèves pour les aider à interagir avec l'aménagement matériel et humain mis en place. Nous avons codé le type d'aménagement du milieu utilisé par l'enseignant à l'aide des catégories préétablies par l'analyse épistémologique (cf. tableau I ci-dessus). De plus, l'ensemble des discours a été transcrit sous la forme de verbatim support à une analyse lexicale (Bardin, 1977). Cette analyse lexicale a été conduite en recourant au logiciel ALCESTE@ ${ }^{1}$. La méthode correspondante vise à « cartographier les principaux lieux communs d'un discours, les mondes lexicaux, qui sont des traces purement sémiotiques inscrites dans la matérialité même du texte » (Kalapalikis \& Moscovici, 2005, p. 15). L'analyse lexicale opérée, ayant pour but de déterminer l'organisation d'un discours, tient au postulat que le vocabulaire usité traduit une intention de sens chez l'émetteur (Reinert, 1999). Ce vocabulaire est envisagé comme une trace et la redondance lexicale est censée permettre de circonscrire des lieux communs discursifs autorisant une interprétation qui débouche sur les thèmes référentiels correspondants (Kalapalikis, 2003). La procédure ainsi dessinée à grands traits apparaît dès lors en phase avec notre objectif de compréhension des mots-clés utilisés par l'enseignant lorsque celui-ci régule l'activité des élèves : c'est autour d'eux qu'est censé se structurer l'emploi d'un vocable spécifique intéressant les objets de savoirs mis à l'étude lors des leçons de badminton. L'identification de mots-clés, structurant le discours de l'enseignant lorsqu'il régule les interactions des élèves avec les MO devrait nous permettre d'avancer dans la compréhension des modifications du milieu utilisées pour apprendre. En définitive, nous disposerons de deux types de données principales: les MO utilisées au sein des SA et les mots-clés structurant les discours du professeur lorsqu'il est en activité de régulation auprès des élèves.

\footnotetext{
${ }^{1}$ La dénomination de ce logiciel, originellement conçu par Max Reinert, correspond à l'acronyme d'Analyse des Lexèmes Coocurrents dans les Enoncés Simplifiés d'un Texte.
} 


\section{eJRIEPS 35 avril 2015}

Les données ainsi explicitées sont alors à commenter et à interpréter. II s'agit dès lors d'opérer une mise en perspective des données fournies par le programme épistémologique avec celles recueillies au travers du programme didactique. Les résultats, présentés ci-après, sont ainsi centrés sur les $\mathrm{OA}, \mathrm{MO}$ et $\mathrm{Cl}$ repérés au sein de la lecture anticipatrice dans l'enseignement du badminton. Ces données sont ensuite confrontées aux observations recueillies in situ traduites sous la forme d'OA, MO et de mots-clés utilisés dans les discours.

\section{Résultats}

4.1. Résultats du programme épistémologique

La présentation des résultats s'appuie sur une logique de comparaison entre les données fournies par le programme épistémologique et celles obtenues avec le programme didactique. Selon l'axe épistémologique, en accord avec Roure (2013), l'enseignement du badminton est organisé autour d'une adaptation de schémas tactiques et stratégiques des joueurs à des rapports de force différenciés symbolisés par des adversaires singuliers. Le tableau II ci-dessous synthétise l'enseignement du badminton au travers de trois SA emblématiques. Ces SA sont qualifiées d'emblématiques car elles sont représentatives de la grande majorité des SA employées en badminton. Ce sont des formes typiques d'organisation des objets de savoir que nous retrouvons régulièrement dans l'enseignement de cette APSA.

Tableau II. Synthèse de l'enseignement du badminton d'après Roure (2013)

\begin{tabular}{|c|c|c|}
\hline $\begin{array}{l}\text { OA Tactique : exploiter les } \\
\text { espaces libres et libérés }\end{array}$ & $\begin{array}{l}\text { OA Technique : le dégagé de } \\
\text { coup droit }\end{array}$ & $\begin{array}{l}\text { OA Stratéqique : observer les } \\
\text { points forts et faibles de } \\
\text { l'adversaire }\end{array}$ \\
\hline $\begin{array}{l}\text { MO Thèmes: incertitude spatiale } \\
\text { avec un adversaire }\end{array}$ & $\begin{array}{l}\text { MO Zones: sur le terrain avec un } \\
\text { partenaire }\end{array}$ & $\begin{array}{l}\text { MO Thèmes: incertitude } \\
\text { événementielle avec un adversaire }\end{array}$ \\
\hline $\begin{array}{l}\text { La situation oppose deux équipes } \\
\text { de joueurs. Chaque joueur sort du } \\
\text { terrain après chaque frappe et } \\
\text { laisse sa place à un partenaire qui } \\
\text { rentre par une porte disposée sur } \\
\text { le côté du terrain. Le déséquilibre } \\
\text { crée par cette entrée décalée sur } \\
\text { le côté doit être utilisé par les } \\
\text { joueurs pour tenter de marquer le } \\
\text { point en exploitant les espaces du } \\
\text { terrain adverse. }\end{array}$ & $\begin{array}{l}\text { Le joueur est placé avec un } \\
\text { partenaire qui renvoie le volant } \\
\text { dans une zone centrale. Sur } \\
\text { chaque volant, le joueur essaye de } \\
\text { produire des dégagés en } \\
\text { atteignant des zones de plus en } \\
\text { plus éloignées. }\end{array}$ & $\begin{array}{l}\text { Chaque joueur choisit un coup } \\
\text { interdit pour son adversaire parmi } \\
\text { une liste (smash, amorti droit, } \\
\text { etc.). Les joueurs doivent conduire } \\
\text { leur affrontement en tenant compte } \\
\text { de l'interdiction. }\end{array}$ \\
\hline
\end{tabular}




\section{eJRIEPS 35 avril 2015}

En accord avec le panorama dressé ci-dessus, les principes organisateurs de l'enseignement du badminton se structurent autour de trois $\mathrm{Cl}$ : l'adaptation à l'adversaire, la construction d'un projet tactique et la formation d'un joueur réfléchi. Le premier $\mathrm{Cl}$ correspond à une évaluation de l'état du rapport de force combiné à l'adaptation de la réflexion de l'élève aux caractéristiques adverses. Le deuxième s'intéresse à l'analyse du jeu de l'élève et aux moyens permettant de construire des projets de jeu (jouer vite, placé, fort, en finesse...). Quant au troisième, il est basé sur l'adoption d'une démarche réflexive sur le jeu où l'élève cherche à extraire des invariants tactiques ou des représentations fonctionnelles des différents systèmes de jeu. Le tableau III, ci-après, présente les principaux mots-clés participant à la construction de chacun des $\mathrm{Cl}$ ainsi qu'une phrase illustrative utilisée par les professeurs pour justifier leur orientation en matière de SA. Les données et les phrases illustratives sont issues des analyses lexicales conduites avec le logiciel ALCESTEC.

Tableau III. Les concepts intégrateurs structurant l'enseignement du badminton

\begin{tabular}{|l|l|l|}
\hline L'adaptation à l'adversaire & La construction d'un projet tactique & La formation d'un joueur réfléchi \\
\hline $\begin{array}{l}\text { Mots-clés: repérer, tester, } \\
\text { provoquer, équilibre, favorable, } \\
\text { imprévisible, incertitude, réflexion, } \\
\text { schéma, transversal. }\end{array}$ & $\begin{array}{l}\text { Mots-clés : variation, rupture, } \\
\text { régularité, risque, construction, } \\
\text { smash, trajectoire, diagonale, } \\
\text { cible, zone. }\end{array}$ & $\begin{array}{l}\text { Mots-clés : développer, } \\
\text { ressource, stratégie, acquisition, } \\
\text { observation, profil, représentation, } \\
\text { interaction, gestion, } \\
\text { réinvestissement, projet, modèle. }\end{array}$ \\
\hline $\begin{array}{l}\text { Phrase illustrative : « Il s'agit } \\
\text { d'identifier les schémas de jeu de } \\
\text { rapport de force favorable et } \\
\text { réduire les incertitudes ». }\end{array}$ & $\begin{array}{l}\text { Phrase illustrative : L'objectif } \\
\text { est de construire un jeu autour de } \\
\text { la régularité et la variation des } \\
\text { trajectoires pour déséquilibrer } \\
\text { l'adversaire et terminer le point } \\
\text { dans des zones précises du terrain }\end{array}$ & $\begin{array}{l}\text { Phrase illustrativeloppement des ressources } \\
\text { stratégiques passe par } \\
\text { l'observation des adversaires qu'il } \\
\text { est possible de représenter sous la } \\
\text { forme de profils de jeu » }\end{array}$ \\
\hline
\end{tabular}

\subsection{Résultats du programme didactique}

Les données prélevées grâce aux observations de terrain, au sein du programme didactique, seront présentées selon les axes chronogénétiques et mésogénétiques en accord avec le cadre théorique et l'objectif de la recherche. Premièrement, l'analyse des huit séances conduites par le professeur d'EPS fait état d'un projet d'enseignement à l'échelle d'un cycle que nous pouvons apprécier au travers du découpage chronologique des OA employés dans les différentes SA. La figure 1, ci-dessous, représente la répartition des $\mathrm{OA}$ pendant le cycle à partir des trois catégories établies par le chercheur à 


\section{eJRIEPS 35 avril 2015}

partir d'un travail antérieur (cf. p.10-11). Les résultats sont exprimés sous la forme de pourcentages afin de voir facilement la répartition de chacune des catégories sur chaque séance.

\begin{tabular}{|c|c|c|c|c|c|c|c|c|c|}
\cline { 3 - 9 } \multicolumn{1}{c|}{} & S1 & S2 & S3 & S4 & S5 & S6 & S7 & S8 \\
\hline OA Techniques & $100 \%$ & & & & & & & & \\
& $75 \%$ & & & & & & & & \\
& $50 \%$ & & & & & & & & \\
OA Tactiques & $25 \%$ & & & & & & & & \\
& $100 \%$ & & & & & & & & \\
& $75 \%$ & & & & & & & & \\
\hline & $50 \%$ & & & & & & & & \\
OA Stratégiques & $25 \%$ & & & & & & & & \\
& $100 \%$ & & & & & & & & \\
& $75 \%$ & & & & & & & & \\
& $50 \%$ & & & & & & & & \\
\hline
\end{tabular}

Figure 1. Répartition des OA sur un cycle de huit séances

Les résultats montrent clairement que l'enseignant opte pour une logique de cycle basée sur les apprentissages tactiques. Cette entrée tactique prend appui, à partir du milieu du cycle, sur la mise en place de réflexions stratégiques chez les élèves. Enfin, les apprentissages techniques sont utilisés en tant que ressources motrices susceptibles de faire évoluer les projets de jeu tactiques des élèves. En ce sens, la technique ne constitue qu'un moyen au service des desseins tactiques des joueurs. Sur le plan des séances, nous pouvons remarquer que le professeur associe systématiquement deux types d'apprentissage pour structurer les SA. Les associations privilégiées sont de type tactique - technique et tactique - stratégique. Enfin, nous pouvons noter que les séances 5 et 8 présentent la particularité d'associer les trois types d'OA dans les SA. Ces différentes associations s'opérationnalisent de deux manières différentes: soit il s'agit d'un enchainement de SA différentes du point de vue des apprentissages (une SA tactique, puis une SA technique et enfin une SA tactique), soit il s'agit de situations entremêlant plusieurs OA (une SA stratégique, puis une SA tactique et technique et enfin une SA stratégique et tactique). Afin d'illustrer ce premier panorama général, nous présentons dans le tableau IV ci-après, trois SA emblématiques en les situant dans le cycle. Comme lors des résultats précédents, les SA choisies, qualifiées d'emblématiques, représentent la majorité des SA utilisées au cours du cycle de badminton. Ce sont des formes typiques 


\section{eJRIEPS 35 avril 2015}

d'organisation des objets de savoir que nous retrouvons régulièrement dans l'enseignement conduit par le professeur.

Tableau IV. Illustration de trois SA emblématiques d'un cycle de badminton au lycée

\begin{tabular}{|c|c|c|}
\hline Le & Les & Le \\
\hline $\begin{array}{l}\text { OA Tactique : variation des } \\
\text { trajectoires du volant }\end{array}$ & $\begin{array}{l}\text { OA Stratégique : observer le profil } \\
\text { de jeu de l'adversaire }\end{array}$ & $\begin{array}{l}\text { OA Technique : le smash de coup } \\
\text { droit }\end{array}$ \\
\hline $\begin{array}{l}\text { MO Zones: sur le terrain avec un } \\
\text { adversaire }\end{array}$ & $\begin{array}{l}\text { MO Thèmes: incertitude } \\
\text { temporelle avec un adversaire }\end{array}$ & $\begin{array}{l}\text { MO Zones : élève seul avec séries } \\
\text { de volant à renvoyer }\end{array}$ \\
\hline $\begin{array}{l}\text { Le terrain est divisé en plusieurs } \\
\text { zones (zones courtes, longues et } \\
\text { latérales) qui rapportent } 3 \text { points si } \\
\text { les joueurs placent le volant } \\
\text { dedans ou si leur adversaire est } \\
\text { placé dans la zone et fait une } \\
\text { faute. L'objectif est de faire varier } \\
\text { les trajectoires de volant aux } \\
\text { élèves afin de déséquilibrer leur } \\
\text { adversaire pour finir le point dans } \\
\text { une des zones bonus. }\end{array}$ & $\begin{array}{l}\text { étant placé dans deux } \\
\text { configurations différentes : un } \\
\text { joueur n'a que trois frappes } \\
\text { maximum pour finir le point et } \\
\text { l'autre n'a pas le droit de smasher. } \\
\text { Les joueurs inversent les rôles à la } \\
\text { moitié du match. L'objectif est } \\
\text { d'identifier le profil de jeu préféré } \\
\text { de l'adversaire (attaquant ou } \\
\text { défenseur/placeur). }\end{array}$ & $\begin{array}{l}\text { Sur une série de } 10 \text { volants, les } \\
\text { élèves doivent répéter des smashs } \\
\text { en essayant d'atteindre des zones } \\
\text { placées sur le terrain adverse. Les } \\
\text { zones sont placées sur les côtés et } \\
\text { l'objectif est de privilégier le } \\
\text { placement des smashs plutôt que } \\
\text { leur puissance. }\end{array}$ \\
\hline
\end{tabular}

La première situation, qui s'inscrit dans la leçon $n^{\circ} 2$, a pour objectif de développer un projet de jeu articulé autour de la variation des trajectoires du volant. L'enseignant met en place cette entrée tactique en début de cycle avant d'aborder d'autres projets de jeu comme la rupture de l'échange. L'introduction de cet OA tactique relativement tôt dans le cycle peut s'expliquer par le fait que ce projet de jeu peut être déployé par les élèves à partir d'un large choix de techniques. En effet, les variations peuvent consister en une alternance de jeu long et jeu court avec l'utilisation des dégagés et des amortis. Mais nous pouvons également varier les trajectoires en direction en alternant le jeu à droite et le jeu à gauche avec l'utilisation des dégagés et des drives. L'avantage du projet tactique basé sur la variation est qu'il s'adapte à un large panel d'élèves quel que soit leurs ressources techniques actuelles. La deuxième situation centrée sur un apprentissage stratégique intervient au niveau de la leçon $n^{\circ} 5$, une fois que les élèves sont parvenus à mettre en place leurs premiers projets tactiques. Cet ajout stratégique vise avant tout à travailler l'adaptabilité à des adversaires présentant des profils différents. Enfin la dernière situation, orientée sur un travail technique spécifique, intervient en fin de cycle afin d'optimiser les ressources des élèves en lien avec leurs projets tactiques notamment pour ceux qui se sont orientés vers la rupture de l'échange. Ces trois SA témoins de la logique chronogénétique identifiée chez un enseignant en badminton s'appuient sur un 


\section{eJRIEPS 35 avril 2015}

aménagement du milieu dont nous pouvons examiner les évolutions à l'aune de la logique mésogénétique repérée chez ce professeur d’EPS.

La présentation des résultats selon l'axe mésogénétique s'appuie sur deux types de données principales (les MO et les mots-clés structurant les discours du professeur) dont nous tenterons de rendre compte en relation avec les résultats explicités selon l'axe chronogénétique. Dans un souci de cohérence et afin de respecter les limites imposées par l'article, nous présentons deux MO caractéristiques des modifications du milieu utilisées pour apprendre par ce professeur, ces dernières correspondant aux situations expliquées précédemment aux leçons $n^{\circ} 2$ et $n^{\circ} 5$. Ces deux $M O$ seront mises en relation avec les mots-clés structurant les discours de l'enseignant (établis à partir de l'analyse lexicale du logiciel ALCESTE()) et des exemples de phrases utilisées par le professeur pour réguler l'activité des élèves. Le tableau $\mathrm{V}$ ci-contre rend compte de ces résultats.

Tableau V. Illustration de deux MO caractéristiques de la logique mésogénétique

\begin{tabular}{|c|c|}
\hline Leçon $n^{\circ} 2$ & Leçon $n^{\circ} 5$ \\
\hline $\begin{array}{l}\text { OA Tactique : variation des trajectoires du volant } \\
\text { MO Zones : sur le terrain avec un adversaire }\end{array}$ & $\begin{array}{l}\text { OA Stratégique: observer le profil de jeu de } \\
\text { l'adversaire } \\
\text { MO Thèmes: incertitude temporelle avec un } \\
\text { adversaire }\end{array}$ \\
\hline $\begin{array}{l}\text { Mots-clés: variation, construction, trajectoire, } \\
\text { adversaire, position, profondeur, diagonale, cible, } \\
\text { zone. }\end{array}$ & $\begin{array}{l}\text { Mots-clés : repérer, favorable, réflexion, profil, } \\
\text { schéma, rapport de force, adversaire, déséquilibre. }\end{array}$ \\
\hline $\begin{array}{l}\text { Exemples de phrases pour réguler: « Regarde la } \\
\text { position de ton adversaire pour jouer dans une zone } \\
\text { libre »; « Il faut que tu choisisses une manière de } \\
\text { construire ton point, par exemple avec les } \\
\text { diagonales »; « Tes trajectoires se ressemblent trop. } \\
\text { II faut vraiment avoir des trajectoires différentes pour } \\
\text { déstabiliser ton adversaire ». }\end{array}$ & $\begin{array}{l}\text { Exemples de phrases pour réguler: «Quel est le } \\
\text { profil de ton adversaire ? Est-ce qu'il préfère attaquer } \\
\text { ou placer les volants? »; «Quand tu es en position } \\
\text { favorable, il faut tenter de finir le point car tu n'as que } \\
3 \text { frappes en tout »; « Est-ce que tu as réfléchi à un } \\
\text { schéma de jeu contre lui ? ». }\end{array}$ \\
\hline
\end{tabular}

Lors de la leçon $n^{\circ} 2$, le professeur utilise un aménagement du milieu avec des zones matérialisées au sol. L'utilisation de cette MO possède une double fonction : d'une part elle permet aux élèves de progresser vers l'acquisition d'un objet de savoir relatif à la variation des trajectoires du volant (étant donné que les zones matérialisent des impacts de volant permettant de différencier les placements de celui-ci), et d'autre part les zones sont utilisées par l'enseignant pour structurer son discours auprès des élèves lorsqu'il régule leur rapport avec l'aménagement du milieu. II utilise également les zones afin 


\section{eJRIEPS 35 avril 2015}

d'introduire des notions clés dans l'apprentissage du badminton comme les prises d'informations et de décisions. Ces constats sont valables également pour l'illustration prise dans la leçon $n^{\circ} 5$ dans la mesure où le professeur s'appuie sur la mise en place d'un thème de jeu jouant sur l'incertitude temporelle (l'élève n'a que trois frappes maximum pour terminer le point ou alors il n'a pas le droit de smasher) pour faciliter l'acquisition d'un OA stratégique relatif à l'observation d'un profil de jeu chez l'adversaire. Cette MO participe aussi à la structuration de son discours de régulation en centrant l'attention des élèves sur l'adaptation à l'adversaire.

\subsection{Comparaison de la lecture anticipatrice et des données prélevées in situ} La comparaison des résultats issus de la lecture anticipatrice avec les données recueillies lors d'un cycle de badminton fait apparaître de nombreux points de convergence et quelques différences. Tout d'abord, nous pouvons remarquer des similitudes dans les principes organisateurs de l'enseignement du badminton dans la mesure où les deux programmes (épistémologique et didactique) mettent clairement en avant une entrée tactique et stratégique pour les apprentissages des élèves. Même si le professeur d'EPS valorise les apprentissages tactiques lors de son cycle, nous pouvons noter que le choix des $O A$ est légèrement différent par rapport aux données de la lecture anticipatrice. En effet, alors que les OA tactiques privilégiant la rupture de l'échange sont prioritaires dans l'analyse épistémologique, nous nous sommes rendus compte que les résultats prélevés in situ accordaient plus d'importance à la variation des trajectoires, ceci pouvant s'expliquer par le fait que ce projet tactique est plus facilement maitrisable d'un point de vue des techniques employées par les élèves. Le choix des MO fait également apparaître quelques différences avec une utilisation partagée des thèmes de jeu et des zones pour l'enseignant d'EPS tandis que l'analyse épistémologique identifiait les thèmes de jeu comme prioritaires. Sur un plan proche de la logique mésogénétique, des convergences apparaissent nettement surtout dans les mots-clés structurant les discours de l'enseignant lorsque celui-ci régule l'activité des élèves. En effet, une très grande majorité des motsclés utilisés par le professeur sont communs avec ceux identifiés lors de l'analyse épistémologique pour la construction des concepts intégrateurs. Sur ce plan, la logique des trois concepts intégrateurs déduits de l'analyse épistémologique (l'adaptation à l'adversaire, la construction d'un projet tactique et la formation d'un joueur réfléchi) se retrouve dans les discours employés par l'enseignant. Son travail de régulation, qui prend appui sur les $\mathrm{MO}$, s'articule très souvent avec des mots-clés proches de notions comme 


\section{eJRIEPS 35 avril 2015}

les prises d'informations et de décision, la construction de projets de jeu ou encore la capacité des élèves à s'adapter à des adversaires aux profils différents.

En définitive, nous disposons d'une mise en perspective des programmes épistémologique et didactique appelant à une discussion au regard des convergences et des différences pointées précédemment. Discussion à envisager en outre à la lumière de la part du processus de transposition didactique effectué par l'enseignant d'EPS.

\section{Discussion}

L'analyse des phénomènes transpositifs à l'œuvre dans l'enseignement du badminton met en évidence des cohérences entre un programme épistémologique, basé sur un travail de recherche existant (Roure, 2013), et un programme didactique articulé autour de l'observation d'un cycle de badminton en EPS. Nous avons retrouvé dans l'analyse des processus de chronogénèse et de mésogénèse chez un enseignement d'EPS, la lecture anticipatrice donnée par l'analyse épistémologique portant sur l'enseignement du badminton. Les résultats de nos observations, dans le cadre d'une classe de lycée, ont mis en évidence un enseignement prioritairement centré sur des apprentissages tactiques et stratégiques mis en œuvre au sein d'environnements éducatifs variés utilisant des zones et des thèmes de jeu. A ce titre, notre hypothèse de recherche est validée. Néanmoins, au-delà des nombreuses convergences apparues entre la lecture anticipatrice et les données prélevées in situ, certaines différences ont été constatées comme des choix d'objets d'apprentissages spécifiques au professeur d'EPS. Ces décalages entre le programme épistémologique et le programme didactique sont tout à fait normaux si l'on en croit les travaux de Schubauer-Leoni \& Leutenegger (2005), et sont à interpréter à la lumière de la part du processus de transposition didactique effectué par l'enseignant.

En effet, ces décalages sont les témoignages directs des phénomènes transpositifs à l'œuvre dans l'enseignement du badminton. Ils permettent également de renforcer la posture de la TACD qui valorise l'analyse ascendante de la transposition didactique centrée sur l'enseignant. Cette part du processus de transposition didactique, réalisée par le professeur d'EPS, s'opère selon deux grands axes : 1) la conception du badminton et le type d'élève à former au travers de la pratique et 2) les caractéristiques des élèves au regard des différents projets (établissement, EPS et classe). Sur le plan de la conception de l'activité, les choix déclarés par cet enseignant (en référence à son histoire personnelle, son expérience et son expertise) mettent en évidence que le badminton est conçu comme une activité de gestion d'un rapport de force. L'objectif est de développer la capacité à 


\section{eJRIEPS 35 avril 2015}

gérer un affrontement avec un adversaire, c'est-à-dire faire les bons choix tactiques en fonction de certaines possibilités techniques. Cette conception du badminton est portée par la volonté de former un joueur réfléchi qui analyse son contexte de jeu et évalue l'état du rapport de force afin de prendre les décisions les plus judicieuses pour gagner les échanges. L'enjeu de cette conception rejoint le sens culturel des sports de raquette tel que décrit par Roure (2013) : «Le sens culturel des sports de raquette s'axe désormais sur la capacité réflexive des joueurs sur les plans tactiques et stratégiques pour faire face à des rapports de force différenciés. Les raisons d'agir des pratiquants correspondent ainsi aux analyses et réflexions menées sur les choix tactiques de l'adversaire afin de proposer et faire évoluer des projets de jeu en lien avec ses possibilités techniques » (p. 176). Les différences avec l'analyse anticipatrice apparaissent plus nettement dans le choix des objets d'apprentissage eu égard aux caractéristiques des élèves de cette classe de première. Sans rentrer dans les détails de celles-ci, nous pouvons retenir une hétérogénéité des niveaux de pratique chez les élèves, ce qui est relativement habituel dans le contexte de l'EPS. Dès lors, le choix de l'enseignant de valoriser davantage le projet tactique lié à la variation des trajectoires s'explique par le fait que cette entrée tactique permet de fédérer un grand nombre d'élèves ayant des niveaux différents. En effet, ce projet de jeu peut s'exprimer avec une grande variété de techniques (smash, dégagé, amorti, drive) ce qui permet une adaptation aux niveaux des élèves via le travail de régulation opéré par ce professeur. Par ailleurs, ce travail de régulation, analysé au travers des mots-clés structurant les discours de l'enseignant, est en adéquation avec l'analyse épistémologique dans le sens où les notions clés abordées par le professeur se rapprochent des concepts intégrateurs sous-tendant la structuration des savoirs scolaires du badminton (s'adapter à l'adversaire, construire un projet tactique et former un joueur réfléchi).

Ainsi, la part du processus de transposition didactique effectuée par l'enseignant s'exprime davantage sur le choix des objets d'apprentissage plutôt que sur leurs mises en œuvre qui apparaissent similaires aux analyses anticipatrices. Nous pouvons donc en conclure que les phénomènes transpositifs à l'œuvre dans l'enseignement du badminton peuvent être appréhendés au travers de la mise en perspective des programmes épistémologique et didactique. Nous devons toutefois rester mesurés quant à notre travail d'investigation. Les résultats de cette recherche ne sont valables qu'à l'échelle d'une classe de lycée et sont donc à confirmer par de nouveaux travaux menés dans d'autres niveaux du système éducatif (classes de collège, enseignement technologique...). 


\section{eJRIEPS 35 avril 2015}

Néanmoins, nous pouvons souligner la complémentarité des programmes épistémologique et didactique, celle-ci ayant été démontrée dans le cadre de l'enseignement du badminton en EPS. Cette complémentarité met en évidence la nécessité de croiser les approches théoriques au niveau des travaux scientifiques centrés sur l'intervention. Il serait intéressant, à cet égard, de conduire des recherches similaires dans d'autres APSA enseignées en EPS afin d'obtenir des regards différents sur les phénomènes d'enseignement et d'apprentissage.

\section{Conclusion}

L'analyse des phénomènes transpositifs à l'œuvre dans l'enseignement du badminton au lycée a été conduite par une comparaison des données issues d'un travail épistémologique existant avec des données prélevées lors d'un cycle d'EPS. La confrontation de ces deux programmes de recherche (épistémologique et didactique), issus de la théorie de l'action conjointe en didactique, a permis de révéler la complémentarité de ces approches, confirmant ainsi les propos de Schubauer-Leoni \& Leutenegger (2005). En tant que telles, les analyses épistémologiques portées sur les objets de savoir, considérées comme des lectures anticipatrices selon Amade-Escot (2013), s'avèrent très intéressantes pour appréhender l'activité des sujets et leurs rapports évolutifs aux objets de savoirs révélés au travers des analyses didactiques in situ. Les résultats avancés dans cette étude participent à la compréhension de la part du processus de transposition didactique réalisé par l'enseignant. De ce point de vue, nous avons pu conclure que l'activité de transposition des professeurs s'exerce davantage au niveau du choix des objets d'apprentissage et de leurs évolutions au fil du cycle en relation avec deux éléments prioritaires qui sont leur conception de l'activité badminton et les caractéristiques des élèves composant la classe. Toutefois, l'ensemble de ces conclusions n'est valable qu'à l'échelle de l'enseignement du badminton dans une classe de lycée et serait à interroger par d'autres investigations (niveau du collège, autre profil de classe...). Enfin, l'adéquation relativement élevée entre les analyses épistémologiques conduites sur des curricula potentiels (publications professionnelles) et les analyses didactiques menées dans une classe de lycée questionne l'influence de ce genre de publications sur la préparation des leçons des enseignants. A ce titre, l'analyse des curricula potentiels pourrait constituer un axe de travail intéressant pour la formation initiale des professeurs d'EPS. Cette piste gagnerait à être investie davantage, d'autant plus que certains auteurs considèrent les curricula potentiels comme des «lectures professionnelles " (Loizon, 


\section{eJRIEPS 35 avril 2015}

Margnes et Terrisse, 2008) ou encore une «mémoire collective » (Lémonie et Robin (2010).

\section{Références bibliographiques}

Amade-Escot, C. (2013). Potentialité de la théorie de l'action conjointe en didactique (TACD) pour l'analyse des situations d'intervention en APS. eJRIEPS, 30, 82-89.

Amade-Escot, C. \& Loquet, M. (2010). L'approche didactique en EPS et en APSA : analyse de l'action conjointe en contextes d'intervention. In M. Musard, M. Loquet et G. Carlier (Dir.) : Sciences de l'intervention en EPS et en sport. (pp. 223-244). Paris : éditions Revue EP.S.

Bardin, L. (1977). L'analyse de contenu. Paris: PUF.

Brousseau, G. (1998). Théorie des situations didactiques. Textes rassemblés et préparés par Balacheff, Cooper, Sutherland, Warfield, Grenoble : la pensée sauvage.

Chevallard, Y. (1985). La transposition didactique. Grenoble : La Pensée Sauvage.

Develay, M. (1992). De l'apprentissage à l'enseignement. Paris : ESF éditeur.

Kalapalikis, N. (2003). L'apport de la méthode Alceste dans l'analyse des représentations sociales. In J.-C. Abric (Dir.) : Méthodes d'étude des représentations sociales. (pp. 147-163). Paris : Editions Erès.

Kalapalikis, N. \& Moscovici, S. (2005). Une approche pragmatique de l'analyse Alceste. Les Cahiers Internationaux de Psychologie Sociale, 66, 15-24.

Lémonie, Y. \& Robin, J-F. (2010). Clinique de l'activité et recherche pour l'intervention en EPS et en sport. In M. Musard, M. Loquet et G. Carlier (Dir.) : Sciences de l'intervention en EPS et en sport. (pp. 87-106). Paris : éditions Revue EP.S.

Loizon, D., Margnes, E. \& Terrisse, A. (2008). Analyse des pratiques d'enseignement du judo en EPS. eJRIEPS, 14, 63-82.

Martinand, J-L. (1986). Connaître et transformer la matière. Berne : Peter Lang.

Musard, M., Robin, J-F., Nachon, M. \& Gréhaigne, J-F. (2008). La « composition sous influences » des curricula : un exemple en acrosport. Science \& Motricité, 64, 6167.

Musard, M., Robin, J-F. \& Caty, D. (2009). La construction des cycles d'acrosport: une démarche originale des enseignants d'EPS. eJRIEPS, 16, 73-85. 


\section{eJRIEPS 35 avril 2015}

Reinert, M. (1999). Quelques interrogations à propos de I'"objet" d'une analyse de discours de type statistique et de la réponse "Alceste". Langage et société, 90, 5770.

Roure, C. (2013). Epistémologie des savoirs scolaires et sens culturel des activités physiques et sportives. Carrefours de l'éducation, 35, 155-170.

Schubauer-Leoni, M.-L. \& Leutenegger, F. (2005). Une relecture des phénomènes transpositifs à la lumière de la didactique comparée. Revue Suisse des sciences de l'éducation, 27(3), 407-429.

Sensevy, G. (2007). Des catégories pour décrire et comprendre l'action didactique. In G.

Sensevy \& A. Mercier (Eds.). Agir ensemble. L'action didactique conjointe du professeur et des élèves, (pp. 13-49). Rennes : Presses Universitaires.

Sensevy, G., Mercier, A. \& Schubauer-Leoni, M. L. (2000). Vers un modèle de l'action didactique du professeur. À propos de la course à 20. Recherche en Didactique des Mathématiques, 20, (3), 263-304. 